\title{
Filterbank Multicarrier and Multicarrier CDMA for Cognitive Radio Systems
}

\author{
Ehsan Azarnasab, Roland Kempter, Neal Patwari and Behrouz Farhang-Boroujeny \\ Department of Electrical and Computer Engineering, \\ University of Utah, 50 South Central Campus Drive, Salt Lake City, Utah, 84112-9206, USA \\ \{azarnasab, kempter, npatwari, farhang\}@ece.utah.edu
}

\begin{abstract}
Recent works in cognitive radio (CR) have suggested multicarrier communication based on orthogonal frequency-division multiplexing (OFDM) for CR networks. In this paper, we propose two alternative multicarrier techniques. The first one, filterbank-based OFDM-OQAM (OFDM-offset quadrature amplitude modulation), permits to maximize the secondary users' spectral efficiency by eliminating the need for guard bands. Also, OFDM-OQAM's filterbank spectral estimator has greater dynamic range than the conventional fast fourier transform in OFDM, which further reduces the probability of undesirable collisions between the secondary users (SU) and primary users (PU). However, OFDM-OQAM still requires an SU base station for distributed sensing. The number and the frequency of required sensing messages for uninterrupted PU operation depends on the randomness of the PU processes. We establish the cost of the CR methodology in the form of signaling bits per bit of payload data and relate our simulation results to the entropy of our PU processes by modeling the behavior of the PUs as finite state Markov chains.

For cases where sensing is not available, when sensing information cannot be conveyed, or when the entropy of the PU processes results in unacceptable sensing-to-payload overhead, we suggest another multicarrier technique, namely multicarrier code division multiple access (MC-CDMA). While MCCDMA does not achieve comparably high spectral efficiency as OFDM-OQAM, the spreading gain of MC-CDMA results in graceful degradation in the case of collisions between PUs and SUs. Furthermore, compared to direct sequence (DS) CDMA, MC-CDMA can exclude narrowband PU interferers locally at the SU receiver, hence improving SU performance. We show that MC-CDMA makes it possible to operate the SU network without distributed sensing and hence without a base station infrastructure and/or a signaling channel.
\end{abstract}

Keywords: Cognitive Radio, CDMA, multicarrier CDMA, OFDM-OQAM, OFDM 


\section{INTRODUCTION}

Cognitive radio (CR) has been proposed to ease the high demand and scarcity of spectrum resources [1]. As opposed to current practice, in which legacy systems have to be discontinued to release spectral resources for new applications, the concept of cognitive radio (or dynamic spectral access) proposes coexistence of the original licensee and new users. The former and the latter are also commonly referred to as the primary users (PU) and secondary users (SU) of the same spectral band. Coexistence is feasible in cases where the characteristics of the licensee result in under-utilization in either the temporal or spectral domain (or both). Early studies into the characteristics of actual spectrum usage by licensed users have shown that under-utilization is common, and large portions of the licensed spectra are rarely used [2], [3].

Researchers have proposed the use of multicarrier modulation, namely OFDM, as a natural candidate for the physical layer of cognitive radio systems [4], [5]. The rationale is that multicarrier systems allow dynamic and efficient harnessing of spectral holes generated by the absent licensee. In addition, the FFT as part of the OFDM demodulator can be used for spectral sensing to detect and avoid PUs. Before we elaborate more on the importance and the functionality of this spectral estimator, a simplification is in order. To shorten notation, we refer to multicarrier solutions with high power spectral density ${ }^{1}$ such as those based on filterbanks or OFDM as narrowband $M C$, or simply NB-MC. Generally, the higher the power spectral density of a secondary user's signal, the greater the potential harm to a primary user's receiver. NB-MC cognitive radio systems must thus be very certain that a channel is free to avoid interference in the form of unwanted PU/SU collisions. Essentially, the secondary users' high-powered subcarrier bands pose significant risk of interference with the primary users, a threat which has to be minimized, if not eliminated, to make the CR-methodology acceptable to a licensee.

Minimization of collision risk is implemented through carrier-sensing, in which a spectral estimator senses the jointly used spectrum before allowing the secondary user's MAC layer to transmit data. As has been shown early in the development of NB-MC for CR systems, such a sensing/collision avoidance method requires the collaborative efforts of a dense SU network to guarantee reliable channel sensing information [5]. Even when such information can be measured, its conveyance to all SU nodes in the network may create interference with the primary users [4], and poses significant reliable multicast challenges as the load in the signaling channel increases [6], [7]. It is thus desirable, to reduce the amount of necessary signaling per unit of payload data in an effort to protect both networks, PUs and

\footnotetext{
${ }^{1}$ Multicarrier solutions with transmission power per subcarrier significantly above the noise floor.
} 
SUs, from interference and congestion, respectively.

One approach to reduce the collision risk in NB-MC is to increase SU spectral efficiency and to improve the accuracy of the SU spectral estimator. A multicarrier technique that tackle both these points is OFDM-OQAM. OFDM-OQAM has originally been proposed by Saltzberg in 1967 [8] and revisited for CR networks recently by Farhang-Boroujeny and Kempter in [9]. OFDM-OQAM improves efficiency by eliminating guardbands, transmitting more data per signaling dimension and hence reducing the channel utilization time. A possible measure of this improvement is the ratio of spectral efficiencies in the SU and PU systems, $\theta=\eta^{(\mathrm{SU})} / \eta^{(\mathrm{PU})}$. In this case, $\eta$ refers to how many bits can be transmitted per channel use. Under the assumption of similar traffic patterns, the higher $\theta$, the less the SU system uses the channel compared to the PUs, reducing the likelihood of interference.

Returning to the issue of the spectral estimator, it is easy to see that the higher the dynamic range, the less likely the SU network to 'miss' a weak PU. The dynamic range refers to the difference between the weakest and the strongest signal which can be detected simultaneously by the estimator. Interestingly, it was shown in [9], that the analysis and synthesis filterbanks used for data transmission in OFDMOQAM can serve as excellent spectral estimators, greatly outperforming the FFT and possibly even the 'optimal' Thomson's Multitaper as proposed by Haykin in [10]. However, even with those improvements, an OFDM-OQAM based CR system still poses a potential risk to the licensee, and needs a central SU base station for relaying of distributed sensing information and SU channel assignment.

Fundamentally, the frequency of channel sensing and the amount of transmitted sensing messages is determined by the properties of the PU processes. Hence, without memory or machine learning in the SU network, the signaling overhead caused by a sensing scheme is lower bounded by the entropy rate $H$ introduced to the channel by the licensees. As a consequence, one can establish a performance measure, $\psi=\frac{\text { bits of signaling }=f(H, \kappa)}{\text { bits of payload }}$, which captures the overhead of the CR methodology in the presence of certain licensees. While out of the scope of this paper, we use $\kappa$ to capture the behavior of specific sensing protocols. For example, in a distributed environment, it may be possible to devise different sensing/signaling protocols, which may approach $H$ to similar degrees, while varying in the amount of signaling information exchanged, thus reducing $\psi$. Before we proceed, we note that finding an 'optimal' $\kappa$, may prove to be challenging, since it in turn depends on $H$ and the tolerable impact of the SUs on the PUs. Hence, in the following, we limit ourselves to determining $H$ from a Markov chain model of our PU processes as a function of PU activity, and relate $H$ to our simulation results in the cases of a fixed sensing per packet generation event ratio of one. To elaborate, in our simulation model, the decision if packets are to be generated is made every $1 \mathrm{~ms}$, which is also the interval in which channel sensing is 
performed. Sensing information, however, will only be transmitted if the SUs' channel state information changed in-between two consecutive soundings.

When a distributed architecture is not available, not usable or simply inefficient - for example, no base station and/or signaling channel is available, the signaling channel is congested, or the high entropy of the PU process leads to high $\psi$ - we propose to use MC-CDMA for CR. MC-CDMA is a combination of CDMA and multicarrier technology [11], where packets are spread in the frequency domain before transmission using the entire frequency band. It follows, that prior to channel usage, the SUs need to determine the time-variant interference temperature [1], [10] for the PUs and choose transmission power, spreading gain as well as transmission rate such that the PU transmissions are not interrupted. Essentially, such a system follows the Ultra-Wide Band (UWB) methodology for Personal Area Networks (PAN). In UWB, transmissions are 'hidden' below the noise floor over a very wide frequency band but coexist with other services. Unlike DS-CDMA (direct sequence CDMA), MC-CDMA allows the SU receiver to exclude interfering PU bands, hence increasing the SUs' Signal to Interference plus Noise Ratio (SINR). This is an important improvement, since SU transmissions occur at very low power and the interference caused by the overlaps with the PUs can have a very detrimental effect on SU performance.

We demonstrate the behavior of OFDM-OQAM and MC-CDMA in the specific example of cellular cognitive radio networks with free-space path loss in the 4.1-4.2 GHz 'mobile' band. In the case of OFDM-OQAM, SU sensing is memoryless and conveyed to and relayed by a central SU base station. Our performance measures are based on the interference temperature as proposed by the FCC [1], [10]. In all three systems, OFDM-OQAM, DS-CDMA and MC-CDMA, whenever SU/PU collisions occur, we calculate the fraction of the overlap along with the mutual interference generated by the SU and the PU packets. This interference results in a decrease of SINR and thus may lead to packet loss. In this paper, we quantify the SINR decrease in a fraction of each packet through a binary approximation. We assume that the FEC code will allow recovery of the complete packet as long as the fraction of the packet that has collided with another transmission remains below a certain percentage. Our investigations include the achievable steady state throughput for both networks.

The paper is organized as follows. At first, we summarize the efforts that have gone into the specification and characterization of NB-MC techniques for CR systems and discuss some latest results in the development of MC-CDMA. We then present our simulation model. Before discussing our simulation results, we relate the amount of transmitted SU sensing messages to the entropy of our PU processes by modeling the PUs' usage of the spectrum as finite state Markov chains. Using simulation results, we then argue that improving the FEC code may not lead to 'invisibility' of the secondary users with 
respect to the PUs. The rationale is that SU/PU packet overlap is caused by PUs that jumped into SU subcarrier bands that have just become active, and is thus likely to last for the entire duration of the packet. Next, we show that our mathematical model of the entropy of the PU processes closely resembles simulation results of the number of transmitted sensing messages in OFDM-OQAM. We conclude the first half of our simulation section showing that thanks to its filterbank spectrum estimator and its high spectral efficiency, our OFDM-OQAM CR network protects the PUs while at the same time providing very high data rates to the SUs.

In the second half, we demonstrate MC-CDMA's ability to effectively 'hide' the CR network from the PUs without the need for distributed sensing, i.e., without the need for a central SU base station. This makes MC-CDMA an excellent physical layer candidate for cases when PU entropy is high (leading to significant sensing overhead), and/or when no signaling channel and/or no SU base station is available. Essentially, MC-CDMA may allow us to notably reduce SU node as well as CR network complexity.

The paper ends with conclusions and an outlook into future research.

\section{Principles of Multicarrier CR COMmunicAtion}

In a series of recent papers, multicarrier communication has been identified as a possible candidate for CR networks [4], [5], [12]. Common to all approaches, CR data transmission is separated into two phases: (1) spectrum sensing and (2) packet transmission. During the spectral sensing phase, unused frequency 'holes' are identified. Then, in the packet transmission phase, a set of the unused subcarriers is modulated to fit into those holes. Of course, the location of the subcarriers has to be updated and verified dynamically, and this information has to be distributed among the SUs. Weiss et al. [5] showed that in order to guarantee minimal interference (that is packet collision rates) between the licensed and the unlicensed users, distributed channel sensing as well as relaying of channel state information by a central SU base station is necessary. Notice that in this approach, it is assumed that the secondary system possesses perfect knowledge of the spectral and temporal location of the licensee. However, as we realize that perfect knowledge is hard to obtain, i.e., comes at the expense of significant signaling overhead, NB-MC systems may dramatically violate the interference temperature limit because of the high power levels of their subcarriers. As a consequence, such systems can lead to severe PU but also SU packet loss. 


\section{A. Filterbank Multicarrier}

While Weiss et al. originally recommended OFDM multicarrier for CR systems, Farhang-Boroujeny and Kempter [9] recently addressed a general problem of OFDM and pointed out its implications for CR networks. The authors showed that in the CR setting, OFDM/FFT can lead to significant errors in the accuracy of the sensing information as well as to tremendous under-utilization of the shared spectral resource. Both effects are caused by the large side lobes of the (FFT-based) OFDM, and methods to improve this situation are expensive. In addition, due to the nature of the OFDM subcarriers, orthogonality cannot be guaranteed if adjacent subcarriers are used by different SUs. As a consequence, the use of long cyclic prefix and windowing techniques has thus been suggested. Unfortunately, these corrective measures lead to significant overhead, and achievable efficiency is in the order of 50\% [9]. Thus, in the rest of this paper, we put OFDM aside and assume a filterbank based spectral estimator and an OFDM-OQAM transceiver for data transmission for our NB-MC scheme. As a physical layer of CR networks, OFDM-OQAM offers the following advantage. The stop-band attenuation of each subcarrier band can be controlled and be made arbitrarily low via the design of the prototype filter. This gives the designer the ability to control the level of interference from SUs to PUs and among SUs. In the remainder, we assume the usage of such a perfect prototype filter which allows us to ignore interference between different subcarriers. Nevertheless, in practice, significant overlap among adjacent subcarriers is inevitable (see Figure 1).

Without getting much into details, it is worth noting that there are three filterbank multicarrier techniques that have been proposed in the literature. Pioneering work on filterbank multicarrier communication techniques was done by Chang [13] and Saltzberg [8] in the mid 1960's. Saltzberg showed that by proper design of a transmit pulse-shape in a multichannel QAM system, and by introducing a half symbol space delay between the in-phase and quadrature components of QAM symbols, it is possible to achieve a baud-rate spacing between adjacent subcarrier channels and still recover the information symbol, free of intersymbol (ISI) and intercarrier interference (ICI). This leads to maximum spectral efficiency. Further progress was made by Hirosaki [14], who showed that the transmitter and receiver part of this modulation method can be implemented efficiently in a polyphase/DFT structure.

In the 1990's, the advancements in digital subscriber line (DSL) technology motivated more activity in the development of other filterbank-based multicarrier communication systems. Early development in this area is an ANSI contribution by Tzannes et al., which was later expanded and called discrete wavelet multitone (DWMT) [15]. In [16], it was shown that DWMT uses cosine-modulated filterbanks which are 
more frequently used for signal compression. The name cosine modulated multitone (CMT) was later adopted for this class of modulators.

Filtered multitone (FMT) is another multicarrier modulation technique specifically developed for DSL applications [17]. As opposed to multicarrier OQAM and CMT, which allow for overlapping of adjacent subcarrier bands, in FMT, subcarrier bands are disjoint. FMT is thus less bandwidth efficient than CMT and OFDM-OQAM. Figure 1 compares the band occupancy of OFDM-OQAM, FMT and CMT.

\section{B. Multicarrier CDMA}

MC-CDMA is a proven and well understood technology [18]-[20]. It can be implemented very elegantly and in a straightforward manner [21] via maximum-ratio combining. As its name suggests, MC-CDMA combines the benefits of the robust transmission and graceful interference properties of DS-CDMA with the flexibility of subcarrier assignments of multicarrier technology. In DS-CDMA, each user uses a unique identification sequence or spreading code of length $N$ for spreading the information sequence of $b_{k}(m)$ bits in the time domain. After the spreading operation, the transmission sequence $s_{k}$ is made up of $N$ chips [22]. Our model of MC-CDMA is similar, albeit the transmissions are spread in the frequency rather than the time domain over different subcarriers. Following the notation in [11], if we denote the $k$ th chip of the spreading code for user $u$ as $c(k, u) \in\{-1,+1\}$, then the transmitted baseband signal for the $m$ th data symbol $b(m)$ is

$$
x(n)=\sum_{n=0}^{N-1} e^{j(2 \pi k n / N) c(k, u) b(m)} .
$$

A schematic of this system is shown in Figure 2. The synthesis and analysis filterbanks can be based on multicarrier OQAM, CMT or FMT. As in the case of NB-MC, for the purpose of simulations in this paper, we have adopted OFDM-OQAM for MC-CDMA also.

Comparable to DS-CDMA, MC-CDMA transmits over the entire available spectrum. However, based on local sensing information, the SU receiver is able to exclude active PU bands, which increases the SUs' SINR. Since this exclusion is performed as part of the demodulator of the MC-CDMA signal on a per symbol basis, our MC-CDMA receiver is able to track PUs which are changing bands frequently without loss of performance. Together with an SU mechanism, which determines the time-variant PU interference temperature and is able to estimate its increase caused by the SU's transmission, MC-CDMA allows to operate an SU network without any distributed sensing. Such an interference measurement technique, although for DS-CDMA CR networks, has been described and analyzed in [23]. 
a) OFDM - OQAM

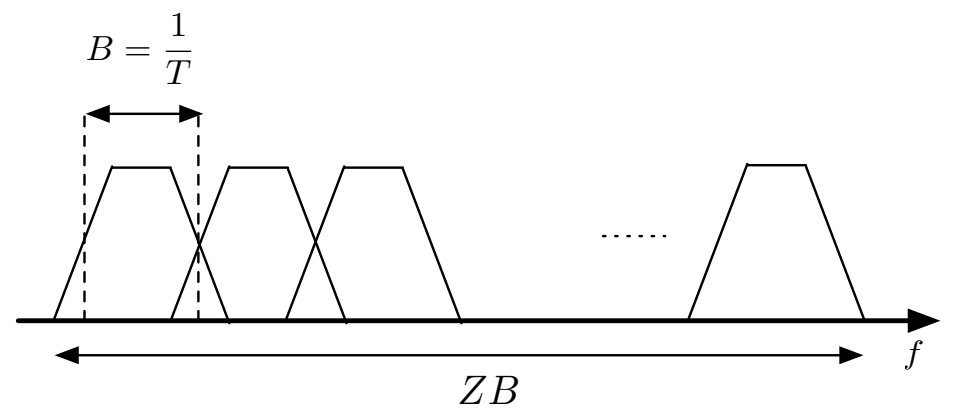

b) FMT

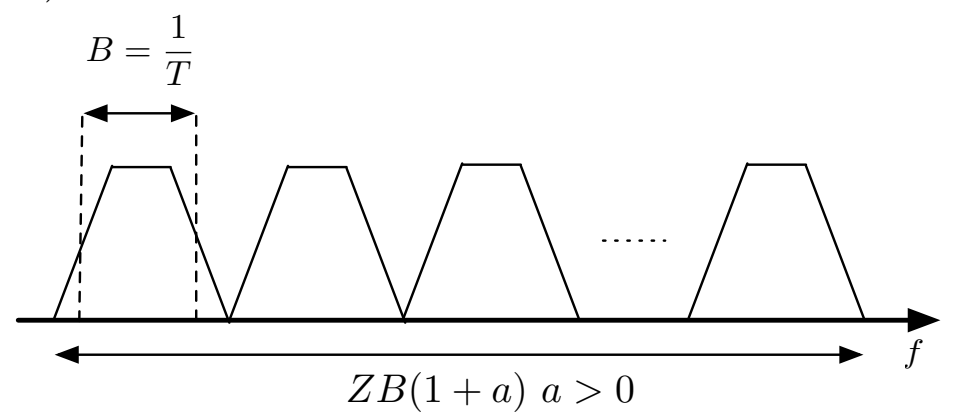

c) $\mathrm{CMT}$

$$
B=\frac{1}{2 T}
$$

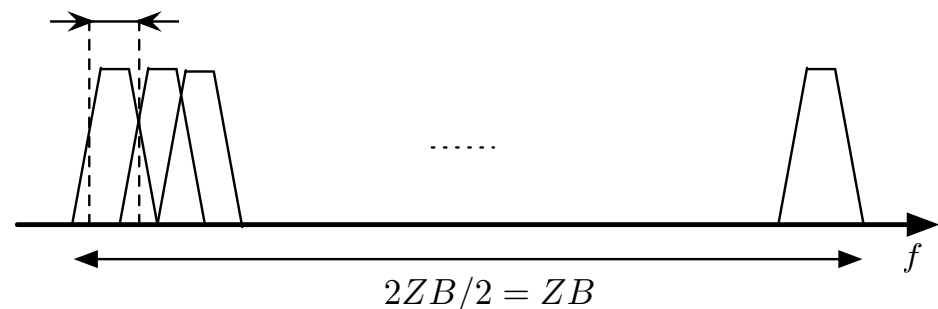

Fig. 1. Subcarrier alignment with a total of $Z$ subcarriers in OFDM-OQAM, filtered multitone (FMT) and $2 Z$ subcarriers in cosine modulated multitone (CMT).

\section{CR Network Simulation, Functionality and Network Parameters}

For our analysis, we implement a CR network simulator in DEVS-Java 3.1 [24]. Wherever possible, the functionality of our CR network follows the IEEE 802.22 standardization group, which is currently developing a standard for a cognitive radio-based PHY/MAC Wireless Regional Area Network (WRAN) [25]. As performance measures, we evaluate the achievable throughput for the PU and SU networks for 
a)
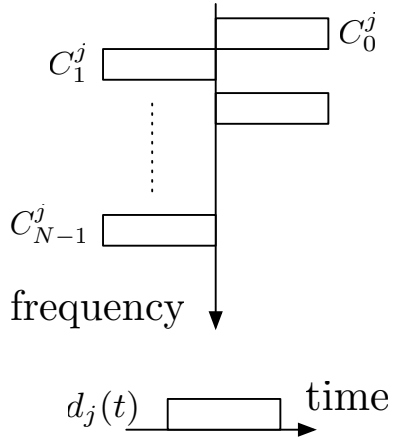

b)

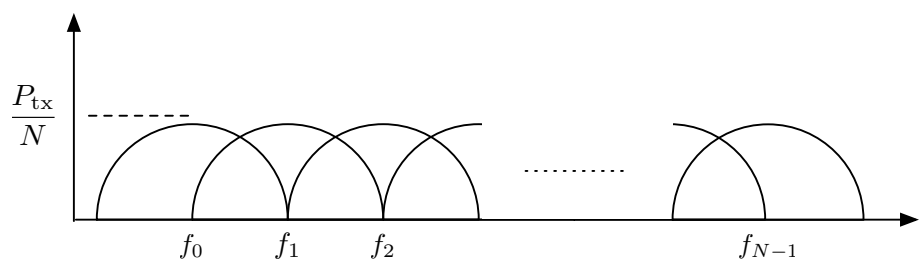

power spectrum of transmitted signal

c)

Transmitter

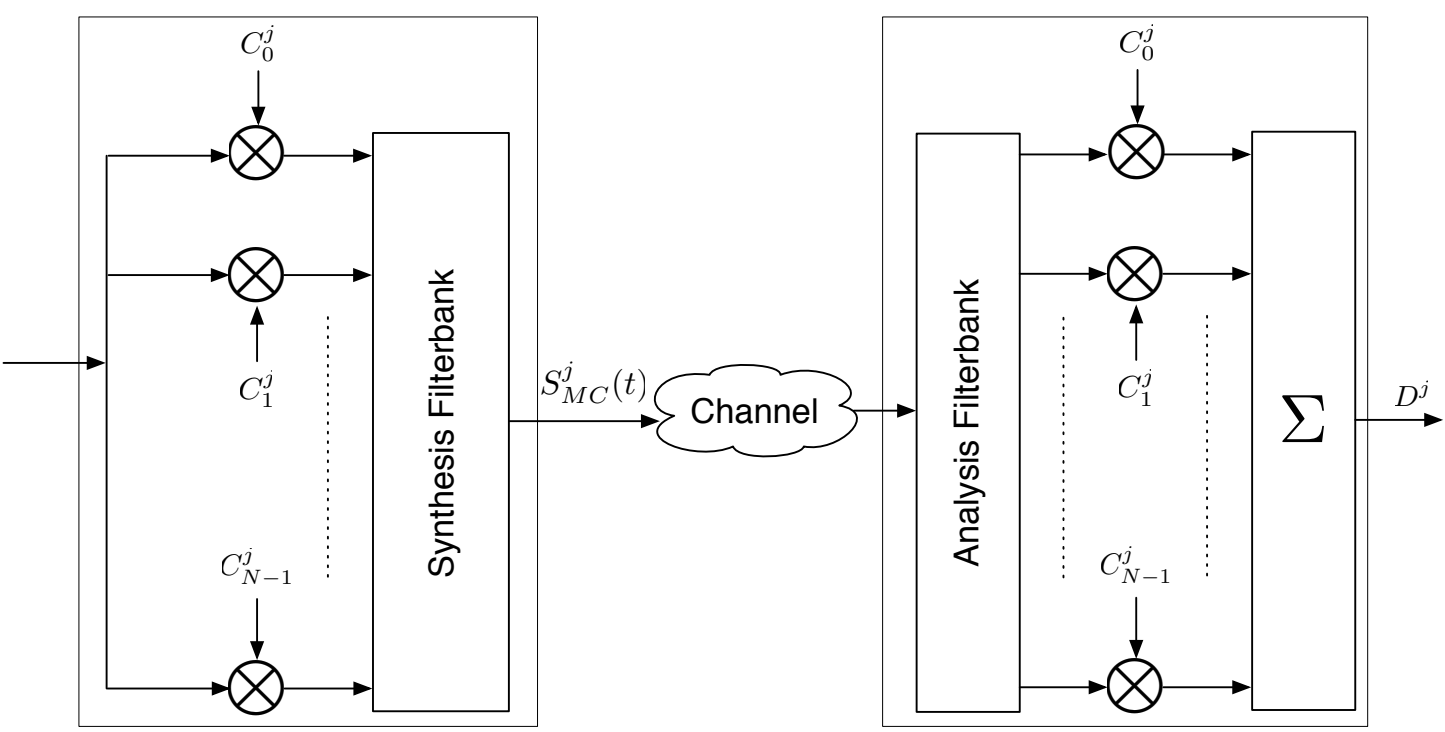

Fig. 2. MC-CDMA transmission scheme [11]. a) spreading operation in the frequency domain; b) power spectrum of transmitted signal over the $N$ subcarriers; c) transceiver system.

our two different multicarrier schemes. In both cases, we assume that our cellular networks operate in the $4.1-4.2 \mathrm{GHz}$ 'mobile' band with free-space path loss. Common simulation parameters are summarized in Table I. The networks each consist of a variable number of PU and SU nodes with associated PU and SU base stations. We further assume that the two base stations are unable to exchange information, as in IEEE $802.22^{2}$. Returning to our set-up, within the two networks, any channel access is coordinated by the corresponding bases, according to the methodology of cellular systems. At this point, we have

${ }^{2}$ This also means that the network of primary users does not have to be a cellular system, and configurations without a PU base are possible analogously. 
not implemented memory or artificial intelligence (AI) in the SU base to be better able to investigate the properties of our two different multicarrier schemes alone. However, a significant reduction of SU/PU interference can be expected as the SU base itself becomes more 'cognitive' and we are currently working on AI methods for PU estimation. For our simulations, we arbitrarily assumed a spectral efficiency of 3 bits/s/Hz with 16 QAM, which requires an effective uncoded SINR, $\gamma$, for successful detection at the PU as well as SU base stations of $\gamma_{\text {req }}=15 \mathrm{~dB}$. To include a safety margin, PU and SU power control sets target SNRs of 20 and $16 \mathrm{~dB}$, respectively, and equalizes transmissions accordingly. It is important to notice that this results in an unequal power situation in the case of PU/SU overlaps whose magnitude depends on the location (that is distance) between the PU and SU base stations.

Simulation time is discretized, and every $1 \mathrm{~ms}$, PUs and SUs generate packets independently according to a Bernoulli process with probability $\operatorname{Pr}_{\text {gen }}, 0<\operatorname{Pr}_{\text {gen }} \leq 1$. Values of $\operatorname{Pr}_{\text {gen }}$ are chosen before an experiment and remain fixed throughout its duration ${ }^{3}$. To better model the behavior of a realistic system, we include queues in the nodes. While for the purpose of this paper, we ignore queuing delays and possible queue instabilities, the queues serve an important function as to when channel requests need to be performed. As packets are generated, they enter the nodes' queues', which triggers channel requests as soon as a certain queue-size, $Q^{(\mathrm{PU})}(t)$ and $Q^{(\mathrm{SU})}(t)$, is achieved. Channel utilization times, $t_{\mathrm{util}}$, can be calculated directly as $t_{\text {util }}=L / R$ using trimodal packet sizes of $L=50, L=500$, and $L=1500$ bytes. Such a packet size distribution is an approximation made for Internet2 backbone traffic [26], but we note that depending on the channel environment, it is possible to fragment larger packets on the MAC layer to make retransmissions less costly. $R$ denotes the bandwidth of the connection and ranges from one subcarrier to eight $(R=1.2 \mathrm{Mbps} \text { to } 9.6 \mathrm{Mbps})^{4}$.

Finally, as soon as a packet transmission is in progress, our simulator tracks the effective SINR at the PU and SU base stations. OFDM-OQAM as well as our PU network both operate with matched filter front ends, which results in

$$
\gamma_{j}^{(\mathrm{mf})}(t)=\frac{P_{j}^{(\mathrm{x})}}{\sigma^{2}+\sum_{\mathcal{O}(t)} P_{i}^{(\mathrm{y})}(t)} .
$$

In (2), $\mathcal{O}(t)$ denotes the set of overlapping PU/SU or SU/PU subcarriers, depending on whether we are detecting an SU or PU packet. Accordingly, the $P_{i}^{(\mathrm{y})}$ are the powers in those subcarriers, $\sigma^{2}$ is the noise variance, and $P_{j}^{(\mathrm{x})}$ is the power of the packet under consideration. Whenever $\gamma_{j}^{(\mathrm{mf})}(t)$ drops below $\gamma_{\text {req }}$,

${ }^{3}$ Of course, more complicated traffic models are possible analogously. The Bernoulli traffic model has been chosen merely to isolate the performance impact of our multicarrier techniques.

${ }^{4}$ As long as channel resources permit, both base stations assign eight subcarriers. 
we mark the number of affected bits as lost. The decision if the corresponding packet can or cannot be recovered is up to our abstracted FEC decoder where we assume a packet to be healthy as long as the collided fraction of the packet is below a certain, variable level. We proceed to describe basic network operations.

\section{A. Network of primary users}

A variable number of PUs along with the PU base station is placed at different (but fixed during each experiment) locations throughout the $1 \mathrm{~km}$ by $1 \mathrm{~km}$ simulation grid. To minimize PU transmission delays, we assume that as soon as $Q^{(\mathrm{PU})}(t)=1$, the PU will perform a channel request and, if successful, transmit the content of its queue with probability $\operatorname{Pr}_{\text {trans }}\left(Q^{(\mathrm{PU})}(t)=1\right)=1$. As long as sufficient channel resources are available, the PU base station always assigns eight consecutive subcarriers to each PU, resulting in an effective channel bandwidth of $8 \times 390,625 \mathrm{kHz}=3.125 \mathrm{MHz}$ and data rate, $R=9.6$ Mbps/PU (see Table I for details). It follows that within our band of $100 \mathrm{MHz}$, we can support a maximum of 32 PUs at maximum speed (respectively any combination of PUs and SUs at maximum speed). From the time the PU was assigned a set of subcarriers, it will transmit at least 100 packets on the same channels before requesting a new set at potentially different, randomly chosen locations. Also defined somewhat arbitrarily, the probability of channel release, $\operatorname{Pr}_{\text {rel }}^{(\mathrm{PU})}$, is linear between the transmission of 100-200 packets, culminating at $\operatorname{Pr}_{\text {rel }}^{(\mathrm{PU})}=1$ after the transmission of 200 packets.

\section{B. Network of secondary users}

In an effort to better understand the behavior of sensing, we ask the SUs to request a channel from the SU base when $Q^{(\mathrm{SU})}(t)=1$. This is necessary to make sure that the SUs are able to perform their "sensing duties'. To elaborate, sensing and data transmission are mutually exclusive, and a full queue may cause an SU to skip one sensing interval if a packet transmission is currently in progress. If the channel resource reservation was successful, the $\mathrm{SU}$ transmits the content of its queue with $\operatorname{Pr}_{\text {trans }}\left(Q^{(\mathrm{SU})}(t)=1\right)=1$ on the assigned subcarriers. Since even perfect sensing cannot avoid interference with PUs which just became active when an SU is already transmitting, see also [4], the application of FEC coding in both schemes appears necessary. In both our systems, we consider the SU network as tolerable to the licensee, as long as it remains 'hardly visible'. For this, we refer to common acceptable packet error rates (PER) in wireless systems. For example, the IEEE 802.20 working group considers a $1 \%$ PER to be acceptable for mobile wireless broadband [27], and even higher rates are tolerated in the more voice-focused UMTS 
TABLE I

COMMON SIMULATION PARAMETERS FOR OFDM-OQAM AND MC-CDMA COGNITIVE RADIO NETWORKS.

\begin{tabular}{|c|c|}
\hline Parameter & Value \\
\hline Operating frequency band & $4.4-4.5 \mathrm{GHz}$ \\
\hline Network coverage area & $1 \mathrm{~km} \times 1 \mathrm{~km}$ \\
\hline Channel model & Free-space path loss \\
\hline Subcarrier bandwidth & $390,625 \mathrm{kHz}$ \\
\hline Number of subcarriers total & 256 \\
\hline Modulation scheme & 16 QAM \\
\hline Spectral efficiency@SINR=15 dB & $3 \mathrm{bits} / \mathrm{s} / \mathrm{Hz}$ \\
\hline Resulting speed per subcarrier & $\approx 1.2 \mathrm{Mbps}$ \\
\hline Guaranteed speed for PUs as long as \# PU $\leq 32$ & 8 subcarriers, resp. $R=9.6 \mathrm{Mbps}$ \\
\hline Min. $E_{\mathrm{b}} / N_{0}$ for succ. detection & $15 \mathrm{~dB}$ \\
\hline $\min P_{\mathrm{tx}}^{(\mathrm{SU})}$ & $16 \mathrm{dBm}$ \\
\hline $\min P_{\mathrm{tx}}^{(\mathrm{PU})}$ & $20 \mathrm{dBm}$ \\
\hline Target power at PU and SU bases (for power control) & $P_{\mathrm{tar}, \mathrm{rx}}^{(\mathrm{SU})}=16 \mathrm{~dB}, P_{\mathrm{tar}, \mathrm{rx}}^{(\mathrm{PU})}=20 \mathrm{~dB}$ \\
\hline Resulting safety margins $\delta$ & $\delta^{(\mathrm{SU})}=1 \mathrm{~dB}, \delta^{(\mathrm{PU})}=5 \mathrm{~dB}$ \\
\hline Receiver sensitivities (SU and PU) & $-85 \mathrm{dBm}$ \\
\hline Dynamic range of SU spectral estimator & $70 \mathrm{~dB}$ \\
\hline Noise level & $-110 \mathrm{dBm}$ \\
\hline \multirow[t]{4}{*}{ Packet size, $L$} & Trimodally distributed: \\
\hline & $\operatorname{Pr}(L=50$ bytes $)=0.5$ \\
\hline & $\operatorname{Pr}(L=500$ bytes $)=0.4$ \\
\hline & $\operatorname{Pr}(L=1500$ bytes $)=0.1$ \\
\hline PU channel release interval & $\begin{array}{l}\operatorname{Pr}_{\mathrm{rel}}^{(\mathrm{PU})}(\# \text { packets }<100)=0, \\
\operatorname{Pr}_{\mathrm{rel}}^{(\mathrm{PU})} \text { lin. between 100-200 packets; } \\
\operatorname{Pr}_{\mathrm{rel}}^{(\mathrm{PU})}(\# \text { packets }=200)=1\end{array}$ \\
\hline Fraction of ECC recoverable bit errors & $2 \%$ of packet size \\
\hline SU and PU packet generation process & Bernoulli processes: $0<\operatorname{Pr}_{\text {gen }} \leq 1$ \\
\hline \# packets in SU queue, $Q^{(\mathrm{SU})}(t)$, before $\mathrm{SU}$ req. channel & $Q^{(\mathrm{SU})}(t)=1 \rightarrow \operatorname{Pr}_{\text {trans }}=1$ \\
\hline \# packets in PU queue, $Q^{(\mathrm{PU})}(t)$, before PU req. channel & $Q^{(\mathrm{PU})}(t)=1 \rightarrow \operatorname{Pr}_{\text {trans }}=1$ \\
\hline
\end{tabular}


systems [5]. As a compromise, for our paper we assume that an SU network can be considered 'safe' to a licensee, if the introduced packet loss does not exceed $1 \%$ as in 802.20.

1) OFDM-OQAM multicarrier: Only for our OFDM-OQAM SU network, we assume the availability of an orthogonal signaling channel. The channel does not reside within the licensed band and is not subject to packet loss or congestion. While in reality, the availability of such a channel is probably unlikely, we can nevertheless use it to investigate the minimal necessary signaling overhead such that the SUs are nonhazardous for the licensees. Once that minimal sensing interval has been determined, we count the amount of sensing information transmitted on the signaling channel as a function of traffic load and network size. Before transmitting the first channel request, SUs sense the entire band and transmit channel state information to the SU base. To minimize overhead, SUs transmit a one-bit identifier per subcarrier, with a high bit indicating that a subcarrier is currently in use by a PU (resulting in a total of 32 bytes of information per sensing per SU, not including packet overhead). Also, the SUs only send new sensing information if it differs from the previous one. The SU base station then performs a logical OR on the collected distributed sensing information and assigns the subcarriers depending on SU demand. After being assigned a set of subcarriers, the SUs start transmitting their packets and continue sensing according to the pre-defined sensing interval (unless they are busy transmitting). Our investigation into signaling overhead will give us a better insight into the properties of operation of NB-MC in general.

Those considerations aside, operation and data transmission of the SU network is similar to the PU network. As long as sufficient channel resources are available, the SU base assigns eight subcarriers for a total speed of 9.6 Mbps to each SU. As resources dwindle, each new SU will be assigned a fair share of the available subcarriers for a reduced speed. Since the transmission power in each of the SU but also PU subcarriers is significant, any overlap needs to be avoided to reduce the negative impact of the SUs on the PUs but also vice versa. The SU network tries to achieve this through frequent carrier sensing, while the PUs (of course) do not try to avoid interfering with the SUs.

2) Multicarrier-CDMA: In MC-CDMA, the maximum spreading gain $N$ available to the SUs depends on the number of active PUs. The recipient of an SU transmission scans the entire spectrum for PUs and excludes the corresponding bands in an effort to increase its SINR. This functionality is part of the MC-CDMA demodulator and performed constantly. As a consequence of the exclusion of possibly any number of subcarriers, orthogonal spreading sequences which only exist for decrements of $N$ which are a power of 2 , cannot be used in our system. We thus resort to random spreading sequences in our implementation of MC-CDMA, which unfortunately leads to non-zero cross correlations and hence multiuser interference between SUs. As our SU multiuser receiver, we turn to the decorrelator, a balanced 
tradeoff between complexity and near-far resistance.

From [28], the SINR after a decorrelation receiver can be calculated as

$$
\gamma_{j}^{(\mathrm{dc})}(t)=\frac{P_{j}^{(\mathrm{x})}\left(1-\frac{K}{N}\right)}{\sigma^{2}+\frac{1}{N} \sum_{\mathcal{O}_{\mathrm{PU}}(t)} P_{i}^{(\mathrm{y})}(t)},
$$

where $K$ is the number of simultaneously active SUs, $\mathcal{O}_{\mathrm{PU}}(t)$ and $P_{i}^{(\mathrm{x})}(t)$ denote the set of PU subcarriers which overlap with the SU with PU power $P_{i}^{(\mathrm{y})}(t)$. Before transmission, the SUs measure the current PU interference temperature limit, following the technique described in [23].

\section{ANAlysis of PU SPECTRAL Information}

Having discussed the simulation setup and before diving into our simulation results, we now quantify the information contained within the PU system's usage of the spectrum over time. Each change in the state of the PU spectral usage is measured and conveyed to the SU base through SU sensing. Since the PU system's occupancy of the spectrum is a random process, by quantifying the entropy rate of this random process, we can determine the minimum data rate needed for the signaling channel to the SU base. Note that for the sake of argument, we put any delay considerations for the signaling messages aside, and investigate the signaling channel's bandwidth requirement as a function of PU behavior, only. Of course, in reality, very strict limitations on signaling delay exist, which need to be considered in the design of NB-MC CR systems. For example, in our system, sensing messages must be delivered to the SU base within one packet generation interval of $1 \mathrm{~ms}$.

A few minor simplifications of the simulated random process allows the PU system's spectral usage to be described as a stationary Markov chain. Denoting this stationary Markov chain as $\left\{X_{i}\right\}$ with stationary distribution $\boldsymbol{\mu}$, its entropy rate $H$ is calculated using [29],

$$
H=-\sum_{i} \mu_{i} \sum_{j} \operatorname{Pr}_{i j} \log _{2} \operatorname{Pr}_{i j}
$$

where $\operatorname{Pr}_{i j}$ is the one-step probability of transition from state $i$ to state $j$.

Rather than assuming that there are 256 channels and each user is assigned 8 consecutive channels, we can assume that there are 32 channel blocks (each 8 channels wide) and each user is assigned one block.

\section{A. Limit as PU Utilization is High}

As $\operatorname{Pr}_{\text {gen }}^{(\mathrm{PU})} \rightarrow 1$, each PU continuously generates packets. However, each PU periodically switches from one channel block to another. Here, we consider the Markov chain in which each state describes 
the channel blocks which are in use at the given time. If we assume 32 channel blocks exist (each block is 8 normal channels) and $J$ number of PUs, then there are $\left(\begin{array}{c}32 \\ J\end{array}\right)$ PU channel assignments. Each state has equal limiting probability, so $\mu=\mu_{i}=\left(\begin{array}{c}32 \\ J\end{array}\right)^{-1}$ for all states $i$.

Periodically, each of the $J$ PUs requests and is assigned a new block. For purposes of analysis using a Markov chain, we assume each PU's reassignment is Bernoulli with probability in each time step of $\lambda_{c}=1 / 150$. Note that while this time step has a different distribution in our simulations (see $\operatorname{Pr}_{\text {rel }}^{(\mathrm{PU})}$ in Table I), it has the same mean time to reassignment. Since $\lambda_{c}$ is low, we neglect cases in which more than one channel is reassigned in a single time step. We assume that each of the $32-J$ empty blocks is equally likely. Since there are $J$ PUs, each state $i$ may transition to one of $J(32-J)$ other states $G_{i}$, where $G_{i}$ is the set of states with block assignments one different than state $i$. Each transition occurs with probability $\operatorname{Pr}_{i j}=\frac{\lambda_{c}}{32-J}$. Note that the probability of staying in state $i, \operatorname{Pr}_{i i}$, is

$$
\operatorname{Pr}_{i i}=1-\sum_{j \neq i} \operatorname{Pr}_{i j}=1-J \lambda_{c}
$$

With these transition probabilities, we can calculate the entropy rate of the Markov chain using (4),

$$
H_{1}=-J(32-J) H\left(\frac{\lambda_{c}}{32-J}\right)-H\left(1-J \lambda_{c}\right)
$$

where $H(x)=x \log _{2} x$. The entropy rate $H$ is shown in Figure 3 as a function of $J$, for three different values of $\lambda_{c}$.

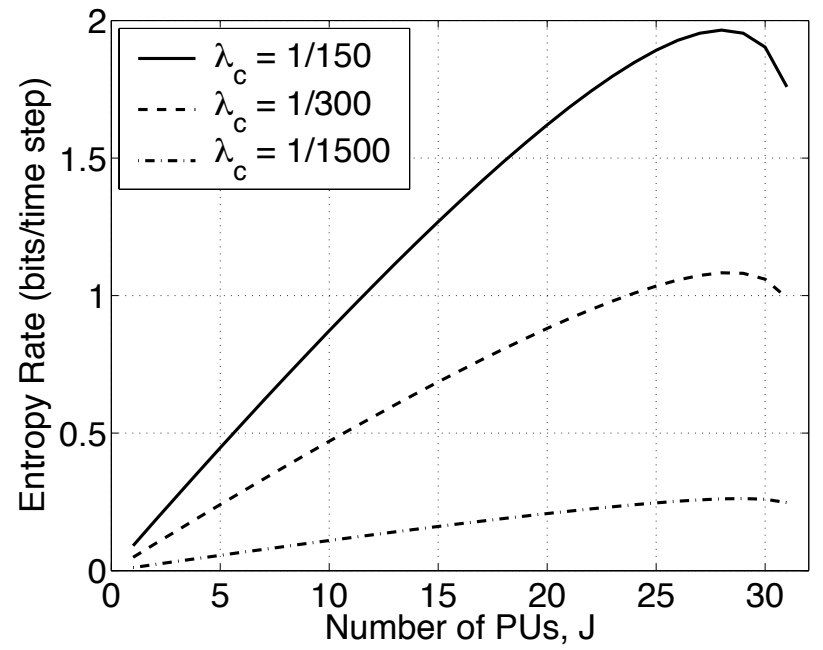

Fig. 3. Calculated entropy rate $H_{1}$ of the high-Prgen $\mathrm{PU}_{\text {gen }}^{(\mathrm{PU})} \mathrm{Ppectral}$ usage random process.

Note that the entropy rate is still high for high $J$ (with a maximum at $J=28$ ). While most channels 
are in use, since each channel switches independently at the same rate $\lambda_{c}$, changes in the spectral usage of the PU system are significant even at high $J$ near 31. It is important to see that for a large range of $J$ (here, $J<10$ ), the entropy rate is nearly linear with $J$. This means that effectively, for low $J$, the primary users are each operating nearly independent random processes. This linearity allows us to proceed with subsequent entropy rate analysis for low $J$.

\section{B. Limit for Few PUs}

The results of Section IV-A indicate that when $J$ is low, we can approximate the PU system spectral usage as $J$ independent random processes, one for each PU. In this section, we approximate each user's random process as a stationary Markov chain. Each state of a PU's Markov chain represents its channel block assignment, and transmission or silence, during the current time step.

We denote state $i_{t}, i=1 \ldots 33-J$, as the state in which the PU is currently transmitting in open channel block $i$. There are $33-J$ open channel blocks because $J-1$ other PUs are each occupying one block, for a total of $33-J+J-1=32$ channel blocks. Further, we denote state $i_{s}, i=1 \ldots 33-J$, as the state in which the PU is assigned open channel block $i$ but is not currently transmitting. It follows that there are a total of $2(33-J)$ states in this Markov chain model.

Assume that the PU has been assigned channel block $i$. The value of $\operatorname{Pr}_{\text {gen }}^{(\mathrm{PU})}$ represents the probability that the PU will be transmitting during the next time step. However, it will (as in Section IV-A) be reassigned a different channel block (among $32-J$ possible blocks) with probability $\lambda_{c}$. Furthermore, from a silent state $i_{s}$, the transition probabilities to other states are identical as when starting at state $i_{t}$. Thus the transition probabilities from state $i_{t}$ or $i_{s}$ are

$$
\begin{aligned}
& \operatorname{Pr}_{i_{s}, j_{t}}=\operatorname{Pr}_{i_{t}, j_{t}}= \begin{cases}\operatorname{Pr}_{\text {gen }}^{(\mathrm{PU})} \lambda_{c} /(32-J), & i \neq j \\
\operatorname{Pr}_{\text {gen }}^{(\mathrm{PU})}\left(1-\lambda_{c}\right), & i=j\end{cases} \\
& \operatorname{Pr}_{i_{s}, i_{s}}=\operatorname{Pr}_{i_{t}, i_{s}}= \begin{cases}0, & i \neq j \\
1-\operatorname{Pr}_{\text {gen }}^{(\mathrm{PU})}, & i=j\end{cases}
\end{aligned}
$$

Since each transmitting state is equally likely, and each silent state is equally likely, the limiting probabilities are $\mu_{i_{t}}=\operatorname{Pr}_{\text {gen }}^{(\mathrm{PU})} /(33-J)$ and $\mu_{i_{s}}=\left(1-\operatorname{Pr}_{\text {gen }}^{(\mathrm{PU})}\right) /(33-J)$, for all $i$. Because of the independence assumption, we assume $J$ independent Markov chains, and the total entropy rate is $J$ times the entropy rate of each chain. Again, using (4), we have that

$$
H_{2}=-J\left[(32-J) H\left(\operatorname{Pr}_{\text {gen }}^{(\mathrm{PU})} \frac{\lambda_{c}}{32-J}\right)+H\left(\operatorname{Pr}_{\text {gen }}^{(\mathrm{PU})}\left(1-\lambda_{c}\right)\right)+H\left(1-\operatorname{Pr}_{\text {gen }}^{(\mathrm{PU})}\right)\right]
$$




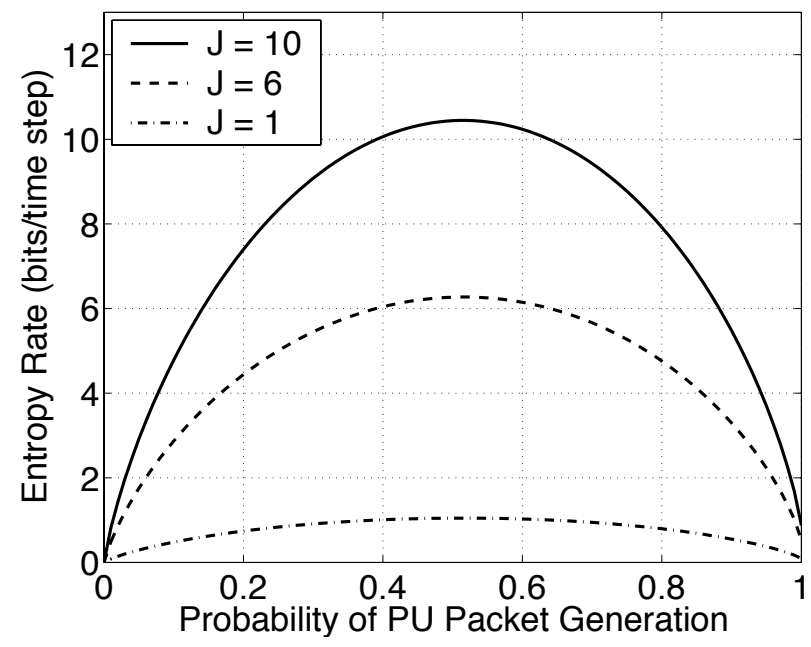

Fig. 4. Calculated entropy rate $H_{2}$ of the low- $J$ PU spectral usage random process.

Figure 4 shows the calculated entropy rate $H_{2}$. Clearly, the entropy rate is not symmetric around $\operatorname{Pr}_{\text {gen }}^{(\mathrm{PU})}=0.5$. In particular, at $\operatorname{Pr}_{\text {gen }}^{(\mathrm{PU})}=1$, the entropy rate is not zero. In fact, the entropy rate is within $0.34 \%$ of the entropy rate $H_{1}$ calculated in Section IV-A for the case of $\operatorname{Pr}_{\text {gen }}^{(\mathrm{PU})}=1$ and $J=1,6$, or 10. This agreement gives confidence in our additional assumption of independent primary users in this section's analysis.

For $\operatorname{Pr}_{\text {gen }}^{(\mathrm{PU})}<1$, the randomness of packet transmission of the PUs creates a significantly higher magnitude of entropy rate. This higher magnitude represents the higher entropy of the transmit/silence of each PU caused by its packet generation process.

\section{Simulation Results}

We proceed to discuss simulation setup and simulation results for OFDM-OQAM, DS-CDMA and MC-CDMA. For both techniques, we simulate different PU and SU network sizes and arrangements. Starting from the maximum setup of ten PUs and ten SUs shown in Figure 5, we remove nodes in decrements of two, starting with node number ten.

\section{A. OFDM-OQAM}

In the first set of experiments, we seek to investigate the positive effect of FEC coding on node performance. We consider two SU and PU activities: $\operatorname{Pr}_{\text {gen }}^{(\mathrm{SU})}=0.2$ with $\operatorname{Pr}_{\text {gen }}^{(\mathrm{PU})}=0.3$, and $\operatorname{Pr}_{\text {gen }}^{(\mathrm{SU})}=0.4$ with $\operatorname{Pr}_{\text {gen }}^{(\mathrm{PU})}=0.6$. Our results have been averaged over 1000 runs and summarized in Table II. As can 


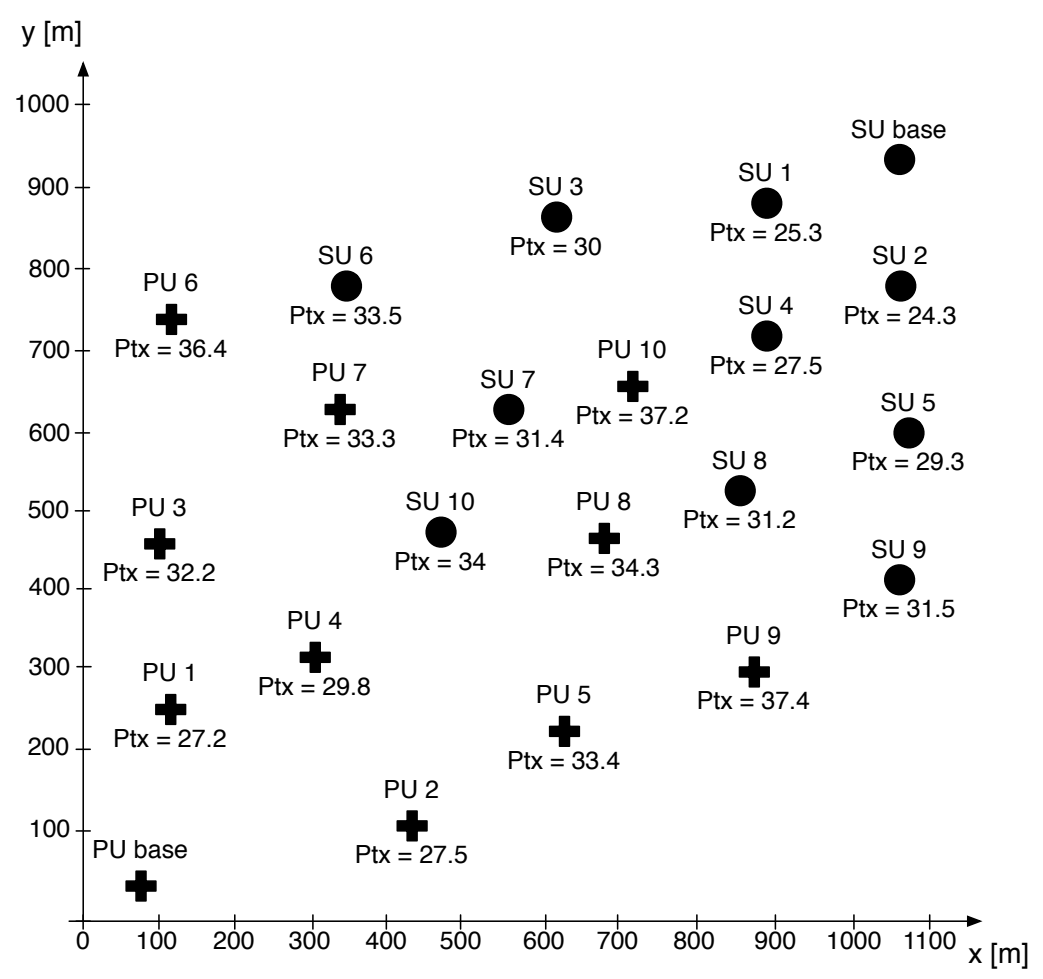

Fig. 5. Maximum-size network configuration of 10 SUs plus SU base (circles) and 10 PUs plus PU base (crosses) with node transmission powers, $P_{\mathrm{tx}}$, in $\mathrm{dBm}$. Smaller network configurations have been realized by removing more and more PUs and SUs, starting with the nodes with the highest numbers.

be seen, improving the FEC to tolerate a larger fraction of lost bits in each packet, does not significantly improve neither PU nor SU performance. The reason is as follows. If the SUs (or PUs for that matter) interfere with the other network, they interfere for a large portion of the affected packet and hence, a significant portion of the PU/SU packet is lost. This happens mostly when PUs jump into SU territory while SUs are transmitting. As a consequence, while the increase in average throughput is not significant as the FEC code gets more powerful, the implementation of such a very powerful code can be costly and reduce the data rate. This means, without SU/PU collaboration, it is likely impossible to avoid SU/PU interference and the PUs will inevitably suffer packet loss. Such losses have to be dealt with on the MAC-layer via packet retransmissions.

In the next step, we take a closer look at the sensing requirements via the number of sensing packets sent as a function of network sizes as well as PU/SU activity. Starting from a minimum sensing interval of $t_{\text {sens }}=1 \mathrm{~ms}$, we keep increasing $t_{\text {sens }}$ up to the point when PU throughput as affected by SU activity 
TABLE II

OFDM-OQAM, IMPACT OF FEC CODE.

\begin{tabular}{|c|c|c|c|}
\hline & \multicolumn{3}{|c|}{ Parameters: 10 PUs, 10 SUs, $\operatorname{Pr}_{\text {gen }}^{(\mathrm{PU})}=0.3, \operatorname{Pr}_{\text {gen }}^{(\mathrm{SU})}=0.2$, sensing interval $=1 \mathrm{~ms}$} \\
\hline $\begin{array}{c}\text { FEC } \\
\text { tolerance in } \%\end{array}$ & $\begin{array}{c}\text { Avg. SU throughput } \\
{[\mathrm{Mbps}]}\end{array}$ & $\begin{array}{l}\text { Avg. PU throughput } \\
{[\mathrm{Mbps}]}\end{array}$ & $\begin{array}{l}\text { \# sensing packets } \\
\text { sent in } 60 \mathrm{~s}\end{array}$ \\
\hline 0 & 9.50 & 9.54 & 57281 \\
\hline 10 & 9.53 & 9.57 & 57291 \\
\hline \multirow[t]{2}{*}{20} & 9.60 & 9.60 & 57255 \\
\hline & \multicolumn{3}{|c|}{ Parameters: 10 PUs, 10 SUs, $\operatorname{Pr}_{\text {gen }}^{(\mathrm{PU})}=0.6, \operatorname{Pr}_{\text {gen }}^{(\mathrm{SU})}=0.4$, sensing interval $=1 \mathrm{~ms}$} \\
\hline $\begin{array}{c}\text { FEC } \\
\text { tolerance in } \%\end{array}$ & $\begin{array}{l}\text { Avg. SU throughput } \\
{[\mathrm{Mbps}]}\end{array}$ & $\begin{array}{l}\text { Avg. PU throughput } \\
{[\mathrm{Mbps}]}\end{array}$ & $\begin{array}{l}\text { \# sensing packets } \\
\text { sent in } 60 \mathrm{~s}\end{array}$ \\
\hline 0 & 9.12 & 9.40 & 94961 \\
\hline 10 & 9.255 & 9.44 & 94955 \\
\hline 30 & 9.38 & 9.48 & 94918 \\
\hline 50 & 9.4 & 9.54 & 95024 \\
\hline
\end{tabular}
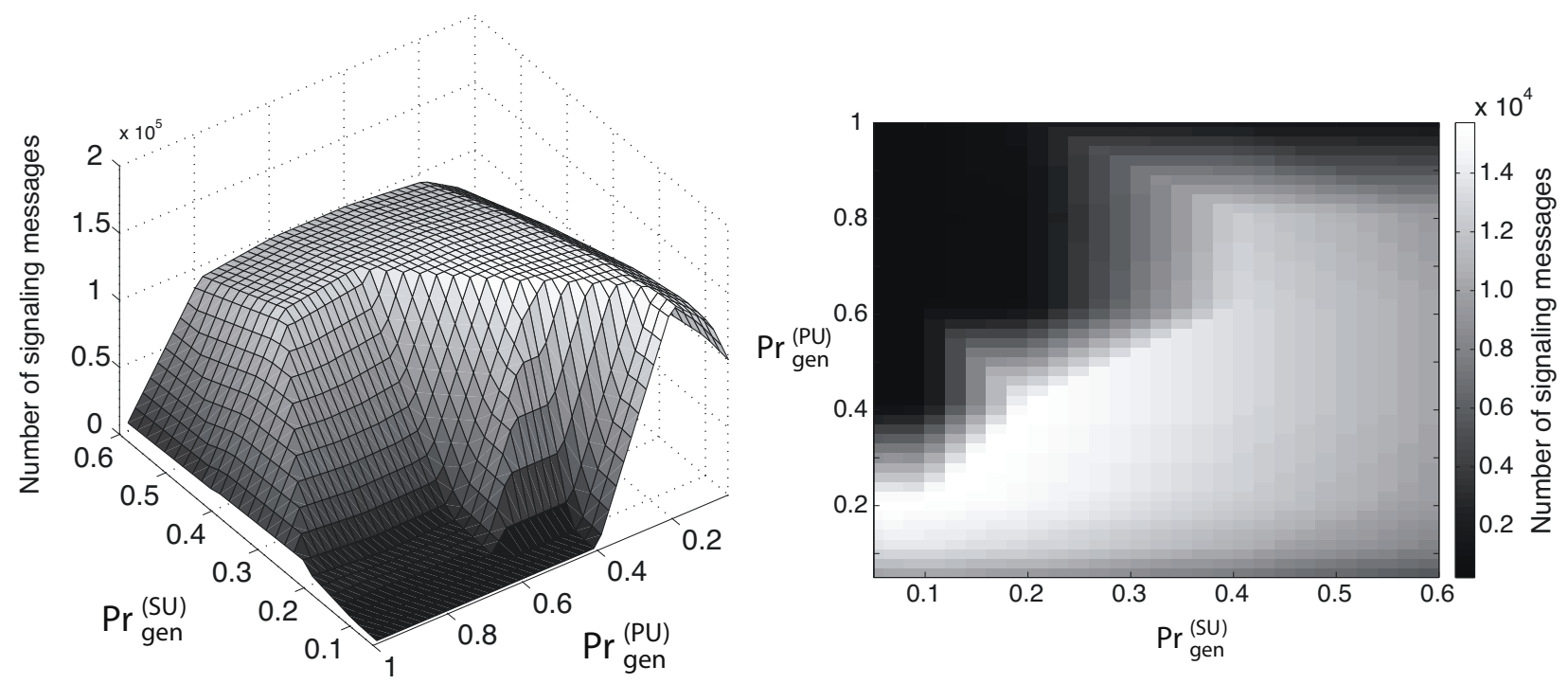

Fig. 6. Number of transmitted signaling messages as functions of PU and SU activity for a network size of 6 PUs and 6 SUs s.th. PU throughput remains at least $99 \%$ optimal. Varying sensing interval, $20 \mathrm{~ms}$ for $\operatorname{Pr}_{\text {gen }}^{(\mathrm{SU})}=0.05$ and $\operatorname{Pr}_{\text {gen }}^{(\mathrm{SU})}=0.1$, in all other cases, sensing interval $=1 \mathrm{~ms}$. 
is reduced by more than $1 \%$. Results are summarized in Figure 6 . In the figure, we show the amount of necessary signaling information being exchanged in the SU network over an interval of $60 \mathrm{~s}$ as PU and SU activity increase. As mentioned previously, SUs only transmit new channel state information, if the channel occupancy as detected by the filterbank spectral estimator changes between measurements. Consequently, the specific value of the sensing interval does not directly determine the amount of information exchanged, but is also a measure of the required SU processing overhead. Returning to the figure, the anomaly in the number of transmitted sensing packets at high PU load and low $\mathrm{SU}$ activity of $0<\operatorname{Pr}_{\text {gen }}^{(\mathrm{SU})}<0.2$ is a result of how our results have been obtained. At low SU activities, the SUs generate only few packets, which means that even if they collide with PUs, the resulting reduction in PU throughput is within our tolerable limit of $1 \%$. Hence, at these low SU activities, our simulator allows a less accurate timing resolution of $20 \mathrm{~ms}$ between sensings (leading to the possibility that SUs 'miss' a PU and thus SU/PU collisions), whereas at all other levels of SU activity, a higher accuracy of $1 \mathrm{~ms}$ was required. Figure 7 shows the number of transmitted sensing messages when we disable this optimization, i.e., with a constant sensing interval of $1 \mathrm{~ms}$. In Figure 7, it can also be seen, that as the SUs become more active, the number of sensing messages is reduced. The reason for this behavior lies in the fact that our SU nodes are unable to perform sensing and packet transmission at the same time. To elaborate, SU channel utilization times lie between $t_{\mathrm{util}}(L=400 \mathrm{bits})=0.42 \mathrm{~ms}$ and $t_{\mathrm{util}}(L=12000 \mathrm{bits})=1.25 \mathrm{~ms}$ with probabilities of occurrence of 50\%, $40 \%$ and $10 \%$. Hence, as $\operatorname{Pr}_{\text {gen }}^{(\mathrm{SU})}$ increases, the number of 12000 bit packets also increases, and thus more and more often, the SUs skip a sensing interval. As a conclusion, this observation underlines our earlier statement, that it is desirable to reduce SU channel utilization times compared to the PUs (respectively increase $\theta$, which can be done easily in OFDM-OQAM, see the discussion in Section II-A), or to implement parallel sensing and packet transmission capabilities to better protect the licensees but also the secondary users. To relate our simulation results to our analytical investigations in Section IV, we note that for any fixed SU activity, Figure 7 matches the shape of the entropy function depicted in Figure 4.

We now investigate if and how the signaling overhead may affect SU network scalability. Figure 8 shows the total number of signaling messages exchanged over 60s for fixed PU and SU activities of $\operatorname{Pr}_{\text {gen }}^{(\mathrm{PU})}=0.6$ and $\operatorname{Pr}_{\text {gen }}^{(\mathrm{SU})}=0.2$ for different PU and SU network sizes. It follows, that the amount of required signaling increases dramatically, as both, the PU and SU network increase beyond six nodes. This behavior has the potential to severely limit the scalability of the SU network and imposes high requirements on the signaling channel. Along these lines, we note that the maximum number of transmitted messages of approximately 160000 in $60 \mathrm{~s}$ shown in Figure 7 translates into $160000 \times 256$ bits $/ 60 \mathrm{~s} \approx 683 \mathrm{Kbps}$ of 


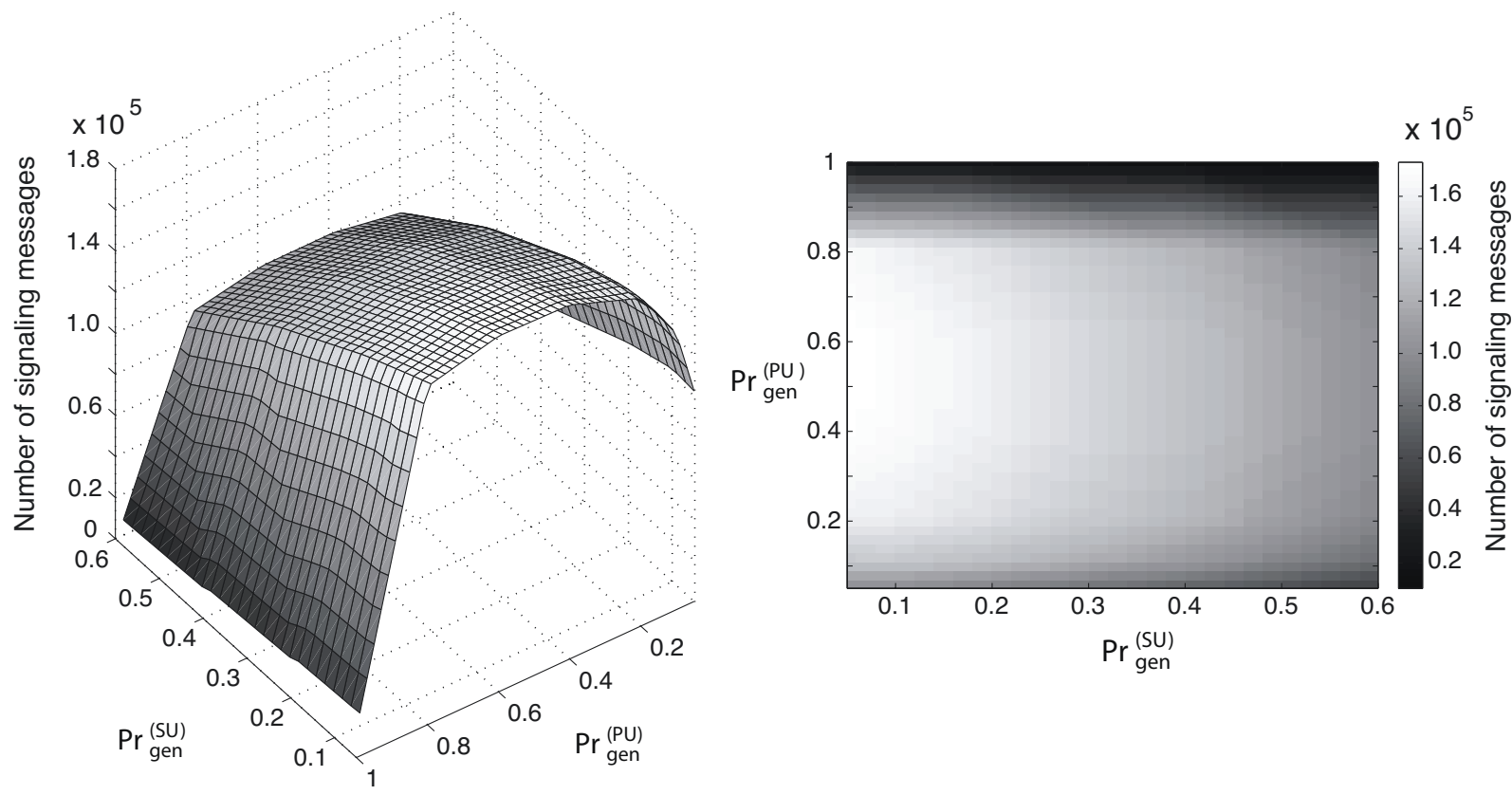

Fig. 7. Number of transmitted signaling messages as functions of PU and SU activity for a network size of 6 PUs and 6 SUs s.th. PU throughput remains at least $99 \%$ optimal. Constant sensing interval of $1 \mathrm{~ms}$.

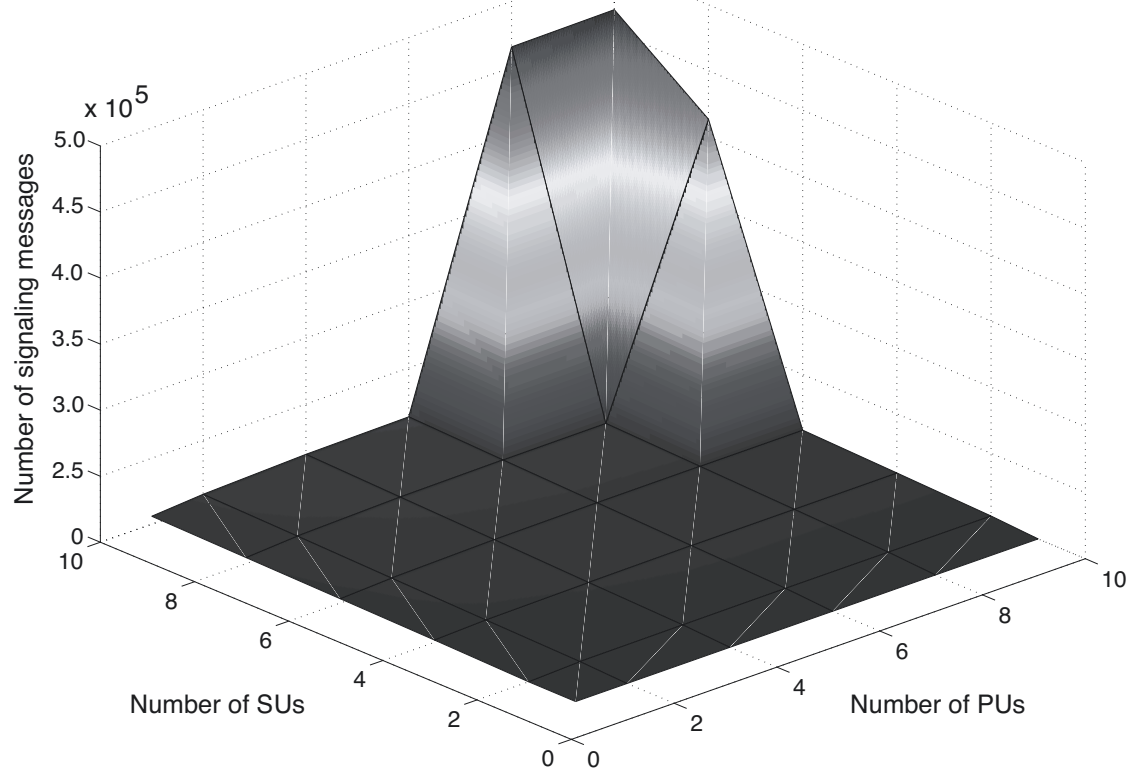

Fig. 8. Number of signaling messages as functions of PU and SU network size for fixed PU and SU activities of $\operatorname{Pr}_{\text {gen }}^{(\mathrm{PU})}=0.6$ and $\operatorname{Pr}_{\text {gen }}^{(\mathrm{SU})}=0.2$, respectively. As the number of PUs and SUs increases beyond six, signaling overhead increases dramatically. 
pure signaling overhead on our optimal, orthogonal signaling channel. Since those messages need to be transmitted in a timely manner (delay has to be less than $1 \mathrm{~ms}$ !), very high requirements for their level of Quality of Service arise. A possible solution to this issue was proposed by Weiss et al. in [5]. There, it was suggested, that the SUs boost the preamble of active PU subcarriers as direct indicators of PU activity. That way, no necessity for a separate signaling channel arises, since channel state information is not conveyed in 'packet-form'. However, while the interference caused by this method (which gets stronger as the SU network increases, since more SUs boost the same preambles) was deemed 'small' in [5], we note that the temporal location of this interference coincides with the beginning of PU packets, which is very critical to packet capture and detection. As a consequence, boosting preambles might not be a very 'PU friendly' method for subcarrier signaling. Comparing our simulation results for the amount of sensing messages transmitted over the signaling channel to our analytical evaluation of the entropy of the PU process in Section IV, we find that the control channel usage, in packets per second, looks similar in shape to Figure 4. In that figure, the maximum spectral information content of the six PUs is approximately 6 bits per packet generation interval of $1 \mathrm{~ms}$. Hence, the minimal amount of information necessary to convey PU behavior is 6 bits $\times 1000 \mathrm{~ms}=6 \mathrm{Kbps}$. While at first glance, it seems that the control channel usage in our simulation is much higher than the entropy rate of the PU processes, in fact, the two results match fairly closely. In contrast to the assumptions which lead to our Markov chain model, in our simulation, the SUs always transmit a full 256 bit subchannel allocation vector, even if only some PU positions changed between observations. Also, opposed to the derivation in Section IV, we did not exploit the fact that in small PU networks, $J<32$, the requirement for the assignment of 8 consecutive PU subcarriers effectively reduces the number of possible PU locations to 32. Furthermore, our analytical results capture the entropy of the PUs as observed by one SU, not by six as in our simulations. If we multiply the results in Section IV by 8 (to compensate for the reduced set of channels) and then by 6 (to account for the presence of 6 SUs which independently sense the channel), our analytical model predicts the transmission of approximately $6000 \times 8 \times 6=288 \mathrm{Kbps}$, which is in the same order of magnitude as our simulation result of $683 \mathrm{Kbps}$. Returning to our measure of the cost of signaling, $\psi$, we find that $\psi$ lies between $\psi_{\text {analytical }}=\frac{288 \mathrm{Kbps}}{9.6 \mathrm{Mbps}}=3 \%$ and $\psi_{\text {sim }}=\frac{683 \mathrm{Kbps}}{9.6 \mathrm{Mbps}}=7 \%$. Remember, that the entropy of the PU system is of course independent of the bandwidth of the SU network and represents the lower bound on the information that needs to be conveyed. Hence, SU link speeds lower than 9.6 Mbps automatically increase $\psi$, reducing the efficiency of the CR methodology. As a conclusion, highly efficient CR physical layers such as OFDM-OQAM may have a bleeding edge over conventional OFDM. On the other hand, we also want to point out, that $\psi$ alone is not a sufficient CR network design parameter. As we will show 
in the following discussion below, the sensing interval should not be reduced below the packet generation interval of the PUs in order to protect the PUs but also the SUs. Hence, sensing messages need to be transmitted to the SU base before this interval expires. Compared to $\mathrm{CR}$ efficiency expressed in $\psi$, this fact is likely to impose a much more severe limitation to the implementation of specific CR networks.

Before we proceed, we want to emphasize, that our OFDM-OQAM system does not have memory or AI, either which may very well lead to a reduction of SU signaling traffic and ease the delay constraints.

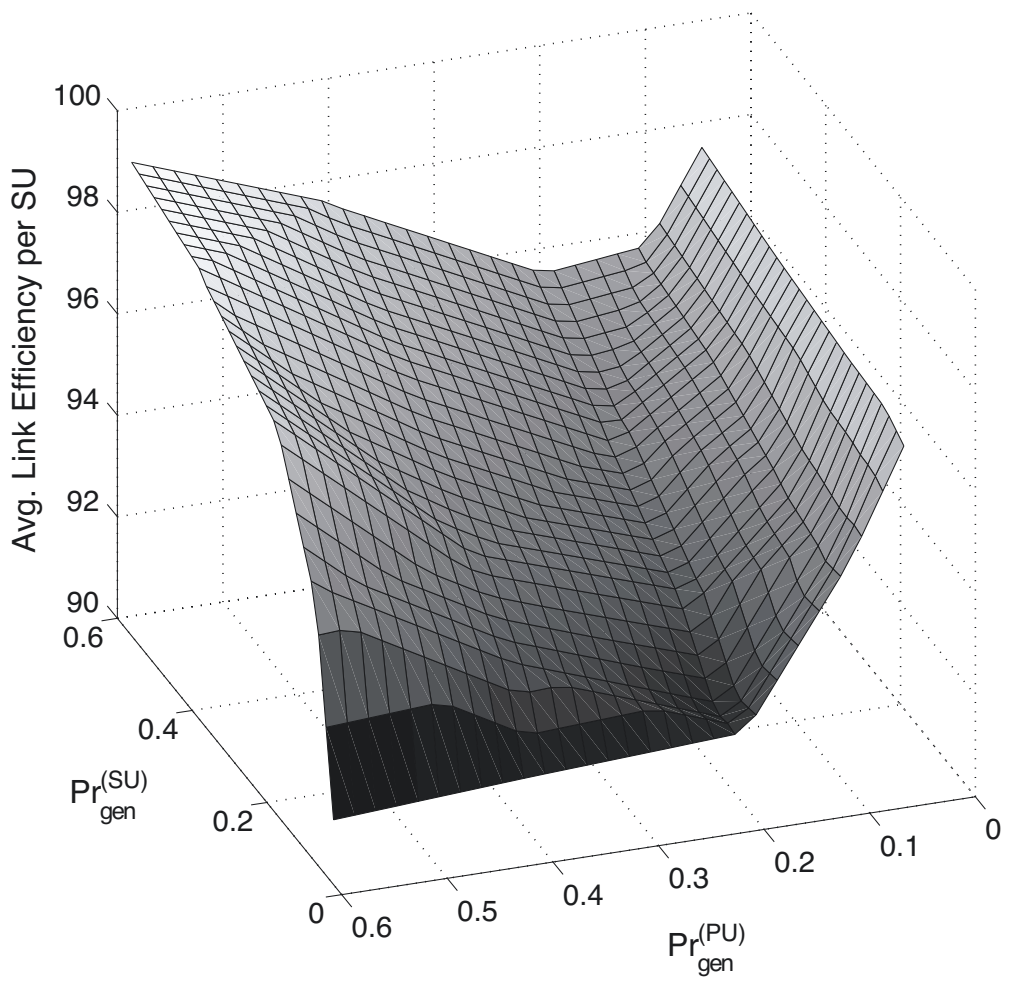

Fig. 9. OFDM-OQAM, average link efficiency per $\mathrm{SU}$ as a function of $\operatorname{Pr}_{\text {gen }}^{(\mathrm{PU})}$ and $\operatorname{Pr}_{\text {gen }}^{(\mathrm{SU})}$. Maximum size network (ten SUs/ten PUs).

We now address the question how PU activity affects achievable SU throughput. As stated previously, the maximum number of nodes that can be supported in our spectrum of $100 \mathrm{MHz}$ is 32 . It follows, that for networks whose total number of SUs+PUs is smaller, SU throughput is close to its maximum of 9.6 Mbps. Consequently, we concentrate on the case with 10 SUs and 10 PUs, resulting in a spectral load factor of $20 / 32=62.5 \%$. Results for average SU link efficiency (100\% corresponds to the full 9.6 Mbps) as a function of PU and SU activity are shown in Figure 9. Starting from minimal PU activity of $\operatorname{Pr}_{\text {gen }}^{(\mathrm{PU})}=0.05$ and very low SU activity, we see a decrease in SU efficiency at a generation probability 
of approximately $\operatorname{Pr}_{\text {gen }}^{(\mathrm{PU})}=0.2$. This point corresponds to the increase of the SU sensing interval to 20 $\mathrm{ms}$ and hence, the dip in SU link efficiency is caused by SU/PU collisions. In summary, while SU/PU collisions may not have a significant impact on PUs if SU transmissions are rare, they have very negative impact on SU throughput. Since SUs send only few packets, losing just some of them has a big negative impact on performance. Thus, if SU performance is also of concern, the sensing interval should not be increased beyond the PU packet generation interval of - in our case $-1 \mathrm{~ms}$.

\section{B. $M C-C D M A$}

In this section, we discuss the performance of our MC-CDMA scheme and compare it to DS-CDMA. To guarantee minimal interference, in both cases, we assume a spreading gain of $N=256$. This is equivalent to an 'unspread' throughput of $1.2 \mathrm{Mbps}$ and results in a safety margin with respect to the PUs of $10 \log _{10}(256) \approx 24 \mathrm{~dB}$. Of course, as the number of active SUs increases, the power on the channel also increases. This requires the systems to either increase $N$ or to reduce the data rates and/or the spectral efficiency. For both our CDMA schemes, we expect SU throughput to drop as we increase the number of active SUs. This is due to an increase of mutual interference and can be compensated via more powerful multiuser receivers, such as the MMSE or iterative detectors [28].

Figure 10 shows average SU throughput with MC-CDMA for a network of six SUs and six PUs, with geographical locations following from Figure 5 after nodes numbered $7-10$ have been removed. The impact of the secondary users on the licensees was within $-1 \%$ of original PU throughput. Before we dive into our simulation results, we want to point out a general positive effect of CDMA in the CR setting. Since CDMA transmissions occur at greatly reduced power levels, the effective interference radius of the SU system is reduced analogously. Referring to the result above, the fact that CDMA transmissions do not affect the PUs, is also a consequence of the specific geographic node locations. As we bring the two networks closer together and disable our interference temperature sensor, we observe an ever increasing negative effect of the SUs on the PUs. However, exponential pathloss works in our favor, and there is a large area in which PUs are indifferent to the SUs. Returning to Figure 10, we see that at low SU and PU activities, MC-CDMA achieves the maximum SU throughput of $1.2 \mathrm{Mbps}$. As activities increase, throughput decreases monotonically to approximately $200 \mathrm{Kbps}$. The decrease in SU performance as a function of PU activity is due to the fact that the effective spreading gain in the SU network reduces as more subcarriers need to be excluded in the SU demodulator.

The superiority of MC-CDMA over DS-CDMA becomes obvious as we move to Figure 11. There, we simulate the performance of DS-CDMA for an identical network setup. Note that the impact of the SU 


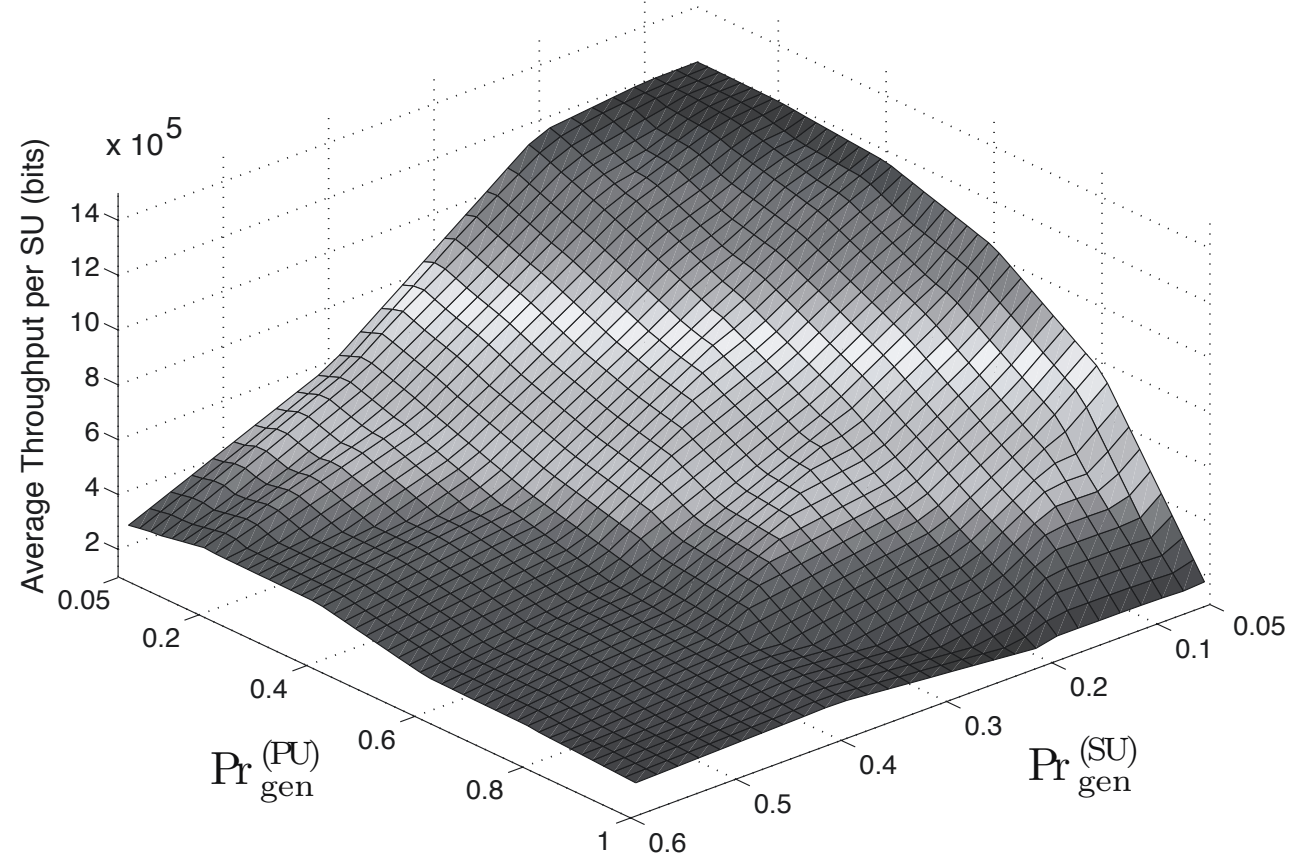

Fig. 10. MC-CDMA, average throughput per SU as a function of SU and PU activity, six SUs and six PUs.

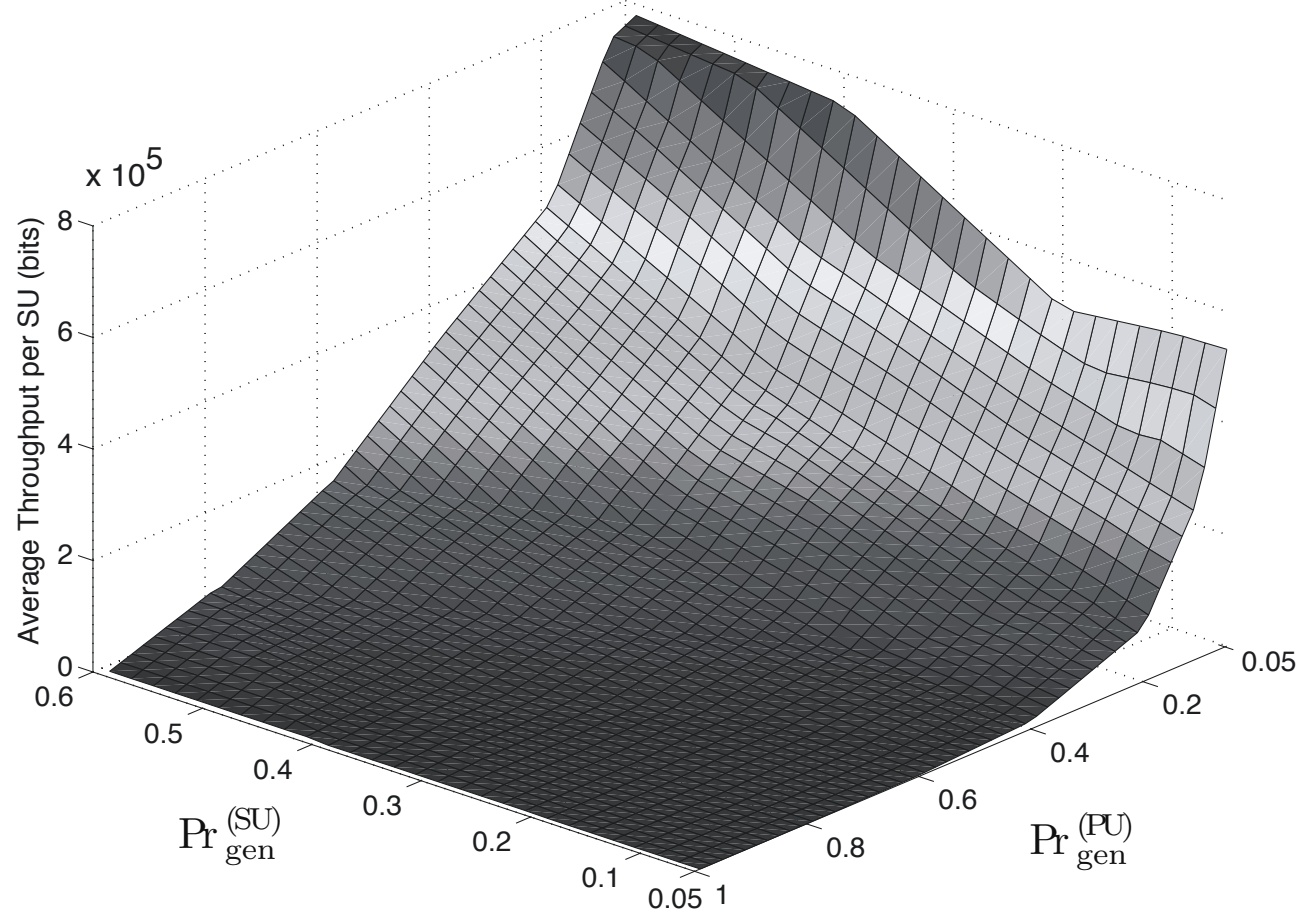

Fig. 11. DS-CDMA, average throughput per SU as a function of SU and PU activity, six SUs and six PUs. 
system on the PUs in terms of SU interference is identical (= zero) in both cases, whereas the outcome for the SUs differs significantly. While with MC-CDMA, SUs are able to 'tune-out' PU interference, such is impossible with DS-CDMA. As a result, SU throughput suffers even at minimal PU activity of $\operatorname{Pr}_{\text {gen }}^{(\mathrm{PU})}=0.05$ and collapses quickly. The fact that SU throughput increases as SUs become more active at low $\operatorname{Pr}_{\text {gen }}^{(\mathrm{PU})}=0.05$, is once more due to the negative effect of losing a few packets out of few transmissions, compared to losing a few packets out of many more. For more information about this effect, we refer back to the discussion about the results shown in Figure 9.

\section{CONCLUSION}

We investigated the performance of three different multicarrier techniques for cognitive radio (CR) networks. For cases when an SU base station and a signaling channel for the transmission of channel sensing information is available, we proposed the application of OFDM-OQAM to CR systems. Based on filterbanks, OFDM-OQAM offers maximum spectral efficiency as well as a very high spectral dynamic range and accuracy in its spectral estimator. We also studied the benefits of strong coding schemes to reduce the possible impact of SUs on PU performance. We found that such measure may not be helpful or significant. To determine the lower limit for the required amount of SU sensing information as a function of the size of the PU network and PU activity, we modeled the spectral behavior of our PUs as finite state Markov chains and calculated its entropy. We showed, that our simulation result of the number of transmitted sensing messages is within the same order of magnitude as our analytical model. As far as CR network scalability is concerned, we noted that as network sizes and SU and PU activities increase, signaling overhead becomes significant, potentially imposing scalability limitations. This is amplified by the very high delay requirements for sensing messages. We showed, that to protect the PUs but also SUs, sensing messages need to be delivered to the SU base station within one PU packet generation, i.e. one PU packet duration interval.

A technique which makes it possible to abandon SU sensing altogether is CDMA for CR. Together with an interference measurement technique, CDMA allows SU transmissions to coexist with the PUs. A commonly used method of CDMA is Direct-Spread CDMA, or DS-CDMA. However, we showed that while not negatively impacting the PUs, DS-CDMA leads to very low SU throughput because of the highpower PU interference on SU transmissions. As a possible solution, we presented multicarrier CDMA (MC-CDMA), which allows the SUs to dynamically exclude PU bands on a per-symbol basis in the SU demodulator. We demonstrated that with MC-CDMA, SU performance greatly exceeds DS-CDMA.

While the investigations carried out in this paper provide important understandings of the behavior and 
characteristics of the OFDM-OQAM and MC-CDMA cognitive radio systems, it also opens up a variety of future research venues. For one, we are currently working on intelligent PU estimation algorithms. In particular, we expect that SU machine learning will be able to reduce the delay and throughput requirements for the signaling channel in OFDM-OQAM, and will allow us to approach the entropy rate of the PUs. Also, we are incorporating a more realistic channel model with fading and multi-path scattering into our simulator.

\section{REFERENCES}

[1] Federal Communications Commission, "Spectrum policy task force," rep. ET Docket no. 02-135, November 2002.

[2] S. Mishra, D. Cabric, C. Chang, D. Willkomm, B. v. Schewick, A. Wolisz, and R. W. Brodersen, "A real time cognitive radio testbed for physical and link layer experiments," Proc. IEEE Symposium on New Frontiers in Dynamic Spectrum Access Networks (DySPAN’05), Nov. 2005.

[3] Shared Spectrum Company, "Spectrum Occupancy Report for New York City during the Republican Convention," http : //www.sharedspectrum.com/?section = measurements, August 30 - September 12004.

[4] T. Weiss and F. Jondral, "Spectrum pooling: an innovative strategy for the enhancement of spectrum efficiency," IEEE Communications Magazine, vol. 42, pp. 8-14, March 2004.

[5] T. Weiss, J. Hillenbrand, A. Krohn, and F. Jondral, "Mutual interference in ofdm-based spectrum pooling systems," IEEE 59th Vehicular Technology Conference, VTC 2004-Spring, vol. 4, pp. 1873-1877, May 17-19 2004.

[6] N. A. Lynch, Distributed Algorithms. No. ISBN 1558603484, Morgan Kaufmann, 1997.

[7] F. D. Towsley, J. Kurose, and S. Pingali, "A comparison of sender-initiated and receiver-initiated reliable multicast protocols," Proceedings of the Sigmetrics Conference on Measurement and Modeling of Computer Systems, ACM Press, pp. 221-230, 1994.

[8] B. Saltzberg, "Performance of an efficient parallel data transmission system," Communications, IEEE Transactions on [legacy, pre - 1988], vol. 15, no. 6, pp. 805-811, 1967.

[9] B. Farhang-Boroujeny and R. Kempter, "Multicarrier communication techniques for spectrum sensing and communication in cognitive radios," submitted to IEEE Journal on Selected Areas in Communications, September 2006, available from http : //www.flux.utah.edu/ rolke/content/FB_CR_CM.pdf.

[10] S. Haykin, "Cognitive radio: brain-empowered wireless communications," IEEE Journal on Selected Areas in Communications, vol. 23, pp. 201-220, February 2005.

[11] S. Hara and R. Prasad, "Overview of multicarrier CDMA," IEEE Communications Magazine, pp. 126-133, December 1997.

[12] F. K. Jondral, "Software-defined radio - basics and evolution to cognitive radio," EURASIP Journal on Wireless Communications and Networking, vol. 3, pp. 275-283, 2005.

[13] R. Chang, "High-speed multichannel data transmission with bandlimited orthogonal signals," Bell Sys. Tech. Journal, vol. 45, pp. 1775-1796, Dec 1966.

[14] B. Hirosaki, "An orthogonally multiplexed qam system using the discrete fourier transform," IEEE Transactions on Communications, vol. 29, pp. 982 - 989, July 1981.

[15] S. Sandberg and M. Tzannes, "Overlapped discrete multitone modulation for high speed copper wire communications," IEEE Journal on Selected Areas in Communications, vol. 13, pp. 1571-1585, Dec. 1995. 
[16] B. Farhang-Boroujeny, "Multicarrier modulation with blind detection capability using cosine modulated filter banks," IEEE Transactions on Communications, vol. 51, pp. 2057-2070, Dec. 2003.

[17] G. Cherubini, E. Eleftheriou, S. Olcer, and J. Cioffi, "Filter bank modulation techniques for very high speed digital subscriber lines," IEEE Communications Magazine, vol. 38, pp. 98-104, May 2000.

[18] S. Kondo and B. Milstein, "Performance of multicarrier ds cdma systems," IEEE Trans. Commun., vol. 44, pp. 238-246, February 1996.

[19] G. K. Kaleh, "Frequency-diversity spread-spectrum communication system to counter bandlimited Gaussian interference," IEEE Trans. Commun., vol. 44, pp. 886-893, July 1996.

[20] M. Tulino, A., L. Li, and S. Verdu, "Spectral efficiency of multicarrier cdma," IEEE Transactions on Information Theory, vol. 51, pp. 479-505, February 2005.

[21] B. Farhang-Boroujeny and C. Furse, "A robust detector for multicarrier spread spectrum transmission over partially jammed channels," IEEE Transactions on Signal Processing, vol. 53, pp. 1038-1044, March 2005.

[22] A. J. Viterbi, CDMA: Principles of Spread Spectrum Communications. Addison-Wesley, 1995.

[23] T. C. Clancy, Dynamic Spectrum Access in Cognitive Radio Networks. PhD thesis, University of Maryland, 2006.

[24] B. P. Zeigler and H. S. Sarjoughian, "Introduction to devs modeling and simulation with java: Developing component-based simulation models," January 2005, the University of Arizona.

[25] The IEEE 802 LAN/MAN Standards Committee, "802.22 WG on Wireless Regional Area Networks (WRANs)," http : //www.ieee802.org/22/.

[26] "Statistics for the Abilene backbone network of Internet2," http : //netflow.internet2.edu/.

[27] The IEEE 802 LAN/MAN Standards Committee, "802.20 WG on Mobile Broadband Wireless Access (MBWA), Draft 802.20 Permanent Document," http : //www.ieee802.org/20/WG_Docs/802.20 - 03 - 15.doc.

[28] S. Verdu and S. Shamai, "Spectral efficiency of CDMA with random spreading," IEEE Transactions on Information Theory, vol. 45, pp. 622-640, March 1999.

[29] T. M. Cover and J. A. Thomas, Elements of Information Theory. Wiley-Interscience, 1991. 